\title{
Chiral Proton Catalysis: A Catalytic Enantioselective Direct aza-Henry Reaction
}

\author{
Benjamin M. Nugent, Ryan A. Yoder, and Jeffrey N. Johnston* \\ Department of Chemistry, Indiana University \\ 800 East Kirkwood Avenue, Bloomington, IN 47405
}

\section{Experimental Section}

Flame-dried (under vacuum) glassware was used for all reactions. All reagents and solvents were commercial grade and purified prior to use when necessary. Tetrahydrofuran (THF) and dichloromethane $\left(\mathrm{CH}_{2} \mathrm{Cl}_{2}\right)$ were dried by passage through a column of activated alumina as described by Grubbs. ${ }^{1}$ The aldimines ${ }^{2}$ and $\operatorname{Pd}(\mathrm{dba})_{2}{ }^{3}$ were prepared as reported in literature. Palladium-mediated aryl amination was executed using a Buchwald protocol. ${ }^{4}$

Thin layer chromatography (TLC) was performed using glass-backed silica gel $(250 \mu \mathrm{m})$ plates and flash chromatography utilized 230-400 mesh silica gel from Scientific Adsorbents. UV light, and/or the use of ceric ammonium molybdate and potassium iodoplatinate solutions to visualize products.

IR spectra were recorded on a Nicolet Avatar 360 spectrophotometer and are reported in wavenumbers $\left(\mathrm{cm}^{-1}\right)$. Liquids and oils were analyzed as neat films on a $\mathrm{NaCl}$ plate (transmission), whereas solids were applied to a diamond plate (ATR). Nuclear magnetic resonance spectra (NMR) were acquired on either a Varian INOVA-400 (400 MHz), VXR-400 (400 MHz), or Varian INOVA-500 (500 MHz) instrument. Chemical shifts are measured relative to residual solvent peaks as an internal standard set to $\delta 7.26$ and $\delta 77.1\left(\mathrm{CDCl}_{3}\right)$. Mass spectra were recorded on a Kratos MS-80 spectrometer by use of chemical ionization (CI). Atlantic Microlabs, GA, performed combustion analyses.

H,Quin-BAM (S1). Pd(dba) $)_{2}(800.0 \mathrm{mg}, 880.0 \mu \mathrm{mol})$, BINAP (1.1 g, $\left.1.76 \mathrm{mmol}\right)$, and $\mathrm{NaO}^{t} \mathrm{Bu}(14.3 \mathrm{~g}, 148.9 \mathrm{mmol})$ were loaded into a round bottom flask in a glove box. Toluene $(250 \mathrm{~mL}, 0.18 \mathrm{M})$ was added to the mixture followed by $1,2-(R, R)$-transdiaminocyclohexane (5.0 g, $43.8 \mathrm{mmol})$. 2-Chloroquinoline (14.3 g, $87.6 \mathrm{mmol})$ was added as a solution in toluene. The reaction was allowed to stir at $80{ }^{\circ} \mathrm{C}$ and monitored

\footnotetext{
${ }^{1}$ Pangborn, A. B.; Giardello, M.A.; Grubbs, R. H.; Rosen, R. K.; Timmers, F. J. Organometallics 1996, 15, 1518-1520.

${ }^{2}$ Kanazawa, A. M.; Denis, J.; Greene, A.E. J. Org. Chem. 1994, 59, 1238-1240.

${ }^{3}$ Rettig, M.F.; Maitlis, P.M. Inorg. Synth. 1992, 28, 110.

${ }^{4}$ Wagaw, S.; Rennels, R.; Buchwald, S. J. Am. Chem. Soc. 1997, 119, 8451-8458.
} 
by TLC. The reaction was then cooled to room temperature, concentrated, and purified by flash column chromatography on silica gel $\left(5 \% \mathrm{Et}_{2} \mathrm{O}\right.$ in hexanes) affording $\mathbf{S 1}$ as a white solid $(13.9 \mathrm{~g}, 86 \%)$ : $[\alpha]_{\mathrm{D}}=+686.2^{\circ}\left(\mathrm{c} 1.0, \mathrm{CHCl}_{3}\right) ; \mathrm{mp} 156-158^{\circ} \mathrm{C} ; \mathrm{R}_{f}=0.13(20 \%$ EtOAc/hexanes); IR (neat) 3410, 3297, 2931, 1618, $1524 \mathrm{~cm}^{-1}$; ${ }^{1} \mathrm{H}$ NMR $(400 \mathrm{MHz}$, $\left.\mathrm{CDCl}_{3}\right) \delta 7.68(\mathrm{~d}, J=8.2 \mathrm{~Hz}, 2 \mathrm{H}), 7.55(\mathrm{~d}, J=8.9 \mathrm{~Hz}, 2 \mathrm{H}), 7.51-7.47(\mathrm{~m}, 4 \mathrm{H}), 7.14(\mathrm{dd}$, $J=7.9,7.0 \mathrm{~Hz}, 2 \mathrm{H}), 6.28$ (d, $J=8.9 \mathrm{~Hz}, 2 \mathrm{H}), 5.86(\mathrm{~s}, 2 \mathrm{H}), 4.12(\mathrm{~s}, 2 \mathrm{H}), 2.37$ (d, $J=12.5$ $\mathrm{Hz}, 2 \mathrm{H}), 1.83-1.81(\mathrm{~m}, 2 \mathrm{H}), 1.52-1.40(\mathrm{~m}, 4 \mathrm{H}) ;{ }^{13} \mathrm{C} \mathrm{NMR}\left(100 \mathrm{MHz}, \mathrm{CDCl}_{3}\right) \delta 169.9$, 157.3, 136.9, 129.6, 127.6, 126.1, 123.5, 121.9, 113.2, 56.3, 33.1, 25.1; HRMS (CI, $\left.\mathrm{CH}_{4}\right)$ Exact mass calculated for $\mathrm{C}_{24} \mathrm{H}_{24} \mathrm{~N}_{4}[\mathrm{M}]^{+} 368.2001$. Found 368.1992.

H,Quin-BAM•HOTf (1). To a solution of BAM in $\mathrm{CH}_{2} \mathrm{Cl}_{2}$ was added freshly distilled trifluoromethanesulfonic acid via syringe at room temperature. The reaction was allowed to stir for 20-30 minutes and was concentrated to afford $\mathbf{1}$ as a white solid: $[\alpha]_{\mathrm{D}}=+450.7^{\circ}$

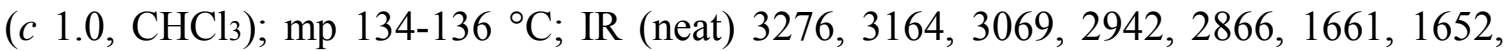
1616, $1532 \mathrm{~cm}^{-1} ;{ }^{1} \mathrm{H}$ NMR (400 MHz, $\left.\mathrm{CDCl}_{3}\right) \delta 8.03$ (br s, $\left.3 \mathrm{H}\right), 7.81$ (m, 4H), 7.61 (dd, $J$ $=8.2,7.0 \mathrm{~Hz}, 2 \mathrm{H}), 7.55(\mathrm{~d}, J=7.9 \mathrm{~Hz}, 2 \mathrm{H}), 7.31(\mathrm{dd}, J=7.6,7.6 \mathrm{~Hz}, 2 \mathrm{H}), 6.82(\mathrm{br} \mathrm{s}$, 2H), $4.19(\mathrm{~m}, 2 \mathrm{H}), 2.18(\mathrm{~d}, J=13.4 \mathrm{~Hz}, 2 \mathrm{H}), 1.86(\mathrm{~d}, J=8.5 \mathrm{~Hz}, 2 \mathrm{H}) 1.72(\mathrm{~d}, J=9.8 \mathrm{~Hz}$, 2H) $1.58-1.50(\mathrm{~m}, 2 \mathrm{H}) ;{ }^{13} \mathrm{C}$ NMR $\left(100 \mathrm{MHz}, \mathrm{CDCl}_{3}\right) \delta 154.2,141.4,132.4,128.7,124.9$, 122.0, 120.6, 118.8, 113.2, 56.7, 31.8, 24.1; HRMS (FAB) Exact mass calculated for $\mathrm{C}_{25} \mathrm{H}_{25} \mathrm{~F}_{3} \mathrm{~N}_{4} \mathrm{O}_{3} \mathrm{~S}[\mathrm{M}]^{+}$369.2079. Found 369.2079.

General Procedure for the Synthesis of Aza-Henry Products (3a-j). A solution of imine (1.0 equiv) and (H,Quin-BAM) ${ }^{H O T f}(0.1$ equiv) in nitromethane or nitroethane $(0.25 \mathrm{M})$ was stirred at $-20^{\circ} \mathrm{C}$ and monitored by GC. The solution was concentrated and purified by flash chromatography $\left(\mathrm{Al}_{2} \mathrm{O}_{3}\right.$ for $\mathbf{3 a - c}$, and silica gel for $\left.\mathbf{3 d - j}\right)$ to furnish the desired product. Enantiomeric excess was determined by chiral HPLC analysis.

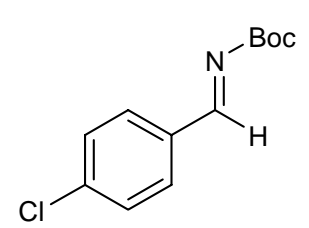

(E)-tert-Butyl 4-chlorobenzylidenecarbamate. Following the Greene protocol, the Schiff base was obtained as a white solid. $\mathrm{Mp}=$ 60-62 ${ }^{\circ} \mathrm{C}$; IR (film) 2976, 1715, 1268, $1154 \mathrm{~cm}^{-1} ;{ }^{1} \mathrm{H}$ NMR (400 $\left.\mathrm{MHz}, \mathrm{CDCl}_{3}\right) \delta 8.85(\mathrm{~s}, 1 \mathrm{H}), 7.87(\mathrm{~d}, J=8.5 \mathrm{~Hz}, 2 \mathrm{H}), 7.47(\mathrm{~d}, J=8.5$ $\mathrm{Hz}, 2 \mathrm{H}) 1.61$ (s, 9H); ${ }^{13} \mathrm{C}$ NMR (125 MHz, $\left.\mathrm{CDCl}_{3}\right)$ ppm 168.5, 162.6, 140.1, 132.8, 131.5, 129.5, 82.8, 28.2; HRMS (CI): Exact mass calcd for $\mathrm{C}_{12} \mathrm{H}_{14} \mathrm{NO}_{2} \mathrm{Cl}[\mathrm{M}]^{+}, 239.0713$. Found 239.0706. 


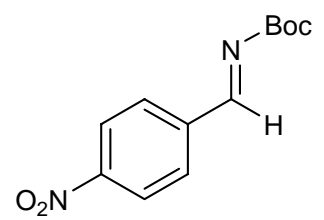

(E)-tert-Butyl 4-nitrobenzylidenecarbamate. Following the Greene protocol, the Schiff base was obtained as a yellow solid. Mp $=102-105^{\circ} \mathrm{C}$; IR (film) 2976, 1707, 1522, 1348, 1157, $852 \mathrm{~cm}^{-1} ;{ }^{1} \mathrm{H}$ NMR $\left(400 \mathrm{MHz}, \mathrm{CDCl}_{3}\right) \delta 8.89(\mathrm{~s}, 1 \mathrm{H}), 8.34(\mathrm{~d}, J=8.7 \mathrm{~Hz}, 2 \mathrm{H})$, $8.10(\mathrm{~d}, J=8.9 \mathrm{~Hz}, 2 \mathrm{H}) 1.62(\mathrm{~s}, 9 \mathrm{H}) ;{ }^{13} \mathrm{C}$ NMR $\left(125 \mathrm{MHz}, \mathrm{CDCl}_{3}\right) \mathrm{ppm} \mathrm{166.5,} \mathrm{162.0,}$ 139.6, 130.9, 124.3, 123.7, 83.5, 28.3; HRMS (CI): Exact mass calcd for $\mathrm{C}_{12} \mathrm{H}_{15} \mathrm{~N}_{2} \mathrm{O}_{4}$ $[\mathrm{M}+\mathrm{H}]^{+}, 251.1032$. Found 251.1037.

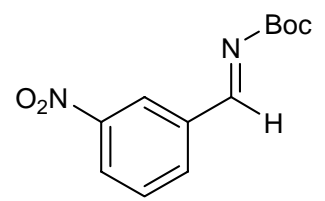

(E)-tert-Butyl 3-nitrobenzylidenecarbamate. Following the Greene protocol, the Schiff base was obtained as a yellow oil. IR (film) 3083, 2978, 2928, 1717, 1533, 1352, 1255, $1155 \mathrm{~cm}^{-1} ;{ }^{1} \mathrm{H}$ NMR (400 MHz, $\left.\mathrm{CDCl}_{3}\right) \delta 8.89(\mathrm{~s}, 1 \mathrm{H}), 8.76(\mathrm{~s}, 1 \mathrm{H}), 8.41(\mathrm{~d}, J=$ $6.7 \mathrm{~Hz}, 1 \mathrm{H}) 8.25(\mathrm{~d}, J=7.7 \mathrm{~Hz}, 1 \mathrm{H}) 7.70(\mathrm{dd}, J=7.9 \mathrm{~Hz}, 1 \mathrm{H}) 1.61(\mathrm{~s}, 9 \mathrm{H}) ;{ }^{13} \mathrm{C} \mathrm{NMR}$ (125 MHz, $\mathrm{CDCl}_{3}$ ) ppm 166.5, 162.0, 148.9, 136.0, 135.4, 130.3, 127.7, 124.8, 83.4, 28.1; HRMS (CI): Exact mass calcd for $\mathrm{C}_{12} \mathrm{H}_{15} \mathrm{~N}_{2} \mathrm{O}_{4}[\mathrm{M}+\mathrm{H}]^{+}$, 251.1032. Found 251.1026.

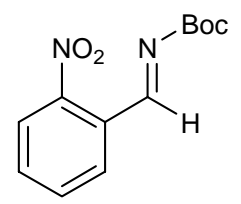

(E)-tert-Butyl 2-nitrobenzylidenecarbamate. Following the Greene protocol, the Schiff base was obtained as a yellow oil. IR (film) 2981, 1721, 1635, 1530, 1369, 1347, 1253, $1155 \mathrm{~cm}^{-1}$; ${ }^{1} \mathrm{H}$ NMR (400 MHz, $\left.\mathrm{CDCl}_{3}\right) \delta 9.21(\mathrm{~s}, 1 \mathrm{H}), 8.17(\mathrm{~d}, J=7.0 \mathrm{~Hz}, 1 \mathrm{H}), 8.07(\mathrm{dd}, J=7.6,0.9 \mathrm{~Hz}$, 1H) 7.71-7.67 (m, 2H) $1.57(\mathrm{~s}, 9 \mathrm{H}) ;{ }^{13} \mathrm{C}$ NMR $\left(125 \mathrm{MHz}, \mathrm{CDCl}_{3}\right) \mathrm{ppm}$ 164.4, 161.8, 150.1, 133.9, 133.2, 130.0, 129.4, 124.9, 83.3, 28.1; HRMS (CI): Exact mass calcd for $\mathrm{C}_{12} \mathrm{H}_{15} \mathrm{~N}_{2} \mathrm{O}_{4}[\mathrm{M}+\mathrm{H}]^{+}$, 251.1032. Found 251.1033.

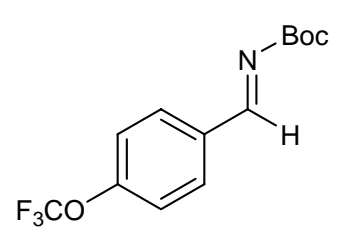

(E)-tert-Butyl

4-(trifluoromethoxy)benzylidenecarbamate.

Following the Greene protocol, the Schiff base was obtained as a clear oil. IR (film) 3330, 2982, 1701, 1507, 1369, 1263, 1223, 1164, $1015 \mathrm{~cm}^{-1} ;{ }^{1} \mathrm{H}$ NMR $\left(400 \mathrm{MHz}, \mathrm{CDCl}_{3}\right) \delta 8.86(\mathrm{~s}, 1 \mathrm{H}), 7.98(\mathrm{~d}, J=$ $8.5 \mathrm{~Hz}, 2 \mathrm{H}), 7.32(\mathrm{~d}, J=8.3 \mathrm{~Hz}, 2 \mathrm{H}) 1.61(\mathrm{~s}, 9 \mathrm{H}) ;{ }^{13} \mathrm{C} \mathrm{NMR}\left(125 \mathrm{MHz}, \mathrm{CDCl}_{3}\right) \mathrm{ppm}$ 167.9, 162.5, 153.1, 132.7, 132.0, 128.0, 121.0, 82.8, 28.1; HRMS (CI): Exact mass calcd for $\mathrm{C}_{13} \mathrm{H}_{15} \mathrm{~F}_{3} \mathrm{NO}_{3}[\mathrm{M}+\mathrm{H}]^{+}$, 290.1004. Found 290.1007.

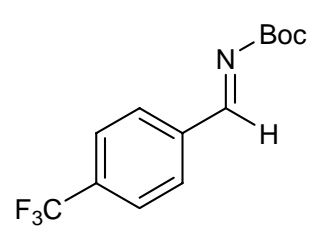

(E)-tert-Butyl

4-(trifluoromethyl)benzylidenecarbamate.

Following the Greene protocol, the Schiff base was obtained as a white solid. IR (film) 3325, 2982, 1696, 1500, 1326, $1168 \mathrm{~cm}^{-1} ;{ }^{1} \mathrm{H}$ NMR (400 MHz, $\left.\mathrm{CDCl}_{3}\right) \delta 8.88(\mathrm{~s}, 1 \mathrm{H}), 8.04(\mathrm{~d}, J=8.2 \mathrm{~Hz}, 2 \mathrm{H})$, 
$7.75(\mathrm{~d}, J=8.2 \mathrm{~Hz}, 2 \mathrm{H}) 1.62(\mathrm{~s}, 9 \mathrm{H}) ;{ }^{13} \mathrm{C} \mathrm{NMR}\left(125 \mathrm{MHz}, \mathrm{CDCl}_{3}\right) \mathrm{ppm} 167.7,162.4$, 137.4, 134.8, 130.4, 126.9, 126.1, 83.1, 28.1; HRMS (CI): Exact mass calcd for $\mathrm{C}_{13} \mathrm{H}_{15} \mathrm{~F}_{3} \mathrm{NO}_{2}[\mathrm{M}+\mathrm{H}]^{+}$, 274.1055. Found 274.1060.

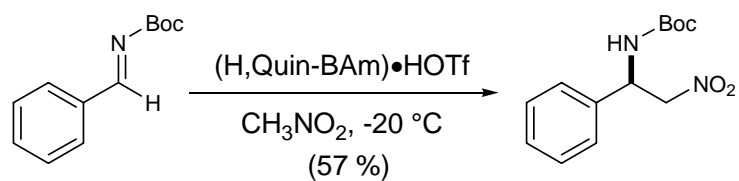

tert-Butyl (R)-2-nitro-1-phenylethylcarbamate (3a). A solution of imine $(100.0 \mathrm{mg}$, $487.0 \mu \mathrm{mol})$ and (H,Quin-BAM) $\cdot \mathrm{TfOH}(25.3 \mathrm{mg}, 48.7 \mu \mathrm{mol})$ in $\mathrm{CH}_{3} \mathrm{NO}_{2}(0.4 \mathrm{~mL}$, $0.25 \mathrm{M}$ ) was stirred at $-20{ }^{\circ} \mathrm{C}$. The solution was concentrated and purified by flash chromatography $\left(\mathrm{Al}_{2} \mathrm{O}_{3}, 20 \%\right.$ ethyl acetate in hexanes) to furnish the product as a white solid $(74.0 \mathrm{mg}, 57 \%$ ) which was determined to be $60 \%$ ee by chiral HPLC analysis (Chiralcel ${ }_{\circledast} \mathrm{AD}, 95: 5$ hexanes: $i-\mathrm{PrOH}, 1 \mathrm{~mL} / \mathrm{min}, \mathrm{t}_{\mathrm{r}}$ (major) $=24.9 \mathrm{~min}, \operatorname{tr}_{\mathrm{r}}$ (minor) $=23.3$ min). $\mathrm{Mp}=116-118{ }^{\circ} \mathrm{C} ; \mathrm{R}_{f}=0.33$ (30\% EtOAc/hexanes); IR (film) 3331, 2984, 1682, $1553 \mathrm{~cm}^{-1}$; ${ }^{1} \mathrm{H}$ NMR $\left(400 \mathrm{MHz}, \mathrm{CDCl}_{3}\right) \delta 7.30-7.40(\mathrm{~m}, 5 \mathrm{H}), 5.37(\mathrm{~m}, 1 \mathrm{H}), 5.29$ (br s, $1 \mathrm{H}), 4.85(\mathrm{~m}, 1 \mathrm{H}), 4.71(\mathrm{dd}, J=12.4,5.2 \mathrm{~Hz}, 1 \mathrm{H}), 1.44(\mathrm{~s}, 1 \mathrm{H}) ;{ }^{13} \mathrm{C}$ NMR $(100 \mathrm{MHz}$, $\mathrm{CDCl}_{3}$ ) ppm 154.9, 137.0, 129.4, 128.9, 126.5, 80.9, 79.1, 53.0, 28.4; HRMS (EI): Exact mass calcd for $\mathrm{C}_{13} \mathrm{H}_{19} \mathrm{~N}_{2} \mathrm{O}_{4}[\mathrm{M}+\mathrm{H}]^{+}, 267.1345$. Found 267.1343.

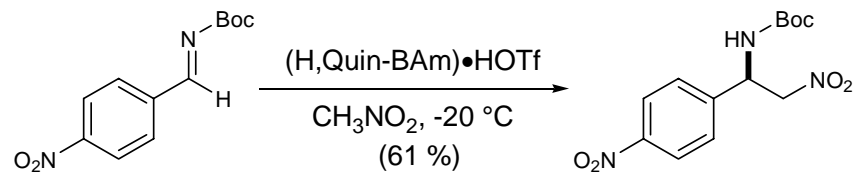

tert-Butyl (R)-2-nitro-1-(4-nitrophenyl)ethylcarbamate (3b). A solution of imine (50.1 mg, $200.0 \mu \mathrm{mol})$ and (H,Quin-BAM) $\cdot \mathrm{TfOH}(10.4 \mathrm{mg}, 20.0 \mu \mathrm{mol})$ in $\mathrm{CH}_{3} \mathrm{NO}_{2}(0.4$ $\mathrm{mL}, 0.25 \mathrm{M})$ was stirred at $-20^{\circ} \mathrm{C}$. The solution was concentrated and purified by flash chromatography $\left(\mathrm{Al}_{2} \mathrm{O}_{3}, 40 \%\right.$ ethyl acetate in hexanes) to furnish the product as a yellow solid $(38.1 \mathrm{mg}, 61 \%)$ which was determined to be $82 \%$ ee by chiral HPLC analysis $\left([\alpha]_{\mathrm{D}}\right.$ $=-14.5^{\circ}$ (c 1.0, acetone) Chiralcel ${ }_{\circledast} \mathrm{AD}, 90: 10$ hexanes: $i-\mathrm{PrOH}, 1 \mathrm{~mL} / \mathrm{min}, \operatorname{tr}$ (major) $=$ $45.3 \mathrm{~min}$, $\operatorname{tr}_{\mathrm{r}}$ minor $\left.)=22.4 \mathrm{~min}\right) . \mathrm{Mp}=132-134{ }^{\circ} \mathrm{C} ; \mathrm{R}_{f}=0.38(60 \%$ EtOAc/hexanes $) ; \mathrm{IR}$ (film) 3353, 2924, 2850, 1701, 1560, $1523 \mathrm{~cm}^{-1} ;{ }^{1} \mathrm{H}$ NMR (400 MHz, $\left.\mathrm{CDCl}_{3}\right) \delta 8.25(\mathrm{~d}, J$ $=8.5 \mathrm{~Hz}, 2 \mathrm{H}), 7.52(\mathrm{~d}, J=8.5 \mathrm{~Hz}, 2 \mathrm{H}), 5.54(\mathrm{br} \mathrm{s}, 1 \mathrm{H}), 5.47(\mathrm{~m}, 1 \mathrm{H}), 4.89(\mathrm{~m}, 1 \mathrm{H}), 4.77$ (dd, $J=13.1,4.3 \mathrm{~Hz}, 1 \mathrm{H}), 1.44$ (s, 9H); ${ }^{13} \mathrm{C}$ NMR (100 MHz, $\mathrm{CDCl}_{3}$ ) ppm 148.2, 144.3, 127.5, 124.6, 81.5, 78.5, 52.3, 28.4; HRMS (EI): Exact mass calcd for $\mathrm{C}_{13} \mathrm{H}_{17} \mathrm{~N}_{3} \mathrm{O}_{6}[\mathrm{M}]^{+}$, 311.1117. Found 311.1116.

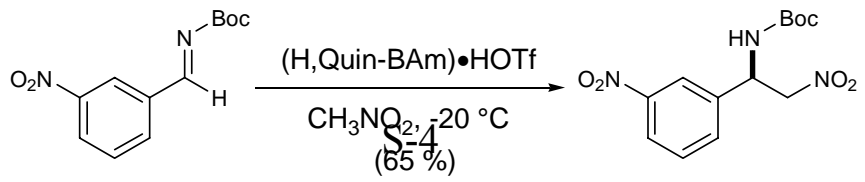


tert-Butyl (R)-2-nitro-1-(3-nitrophenyl)ethylcarbamate (3c). A solution of imine (25.0 $\mathrm{mg}, 100.0 \mu \mathrm{mol})$ and $(\mathrm{H}, \mathrm{Quin}-\mathrm{BAM}) \cdot \mathrm{TfOH}(5.2 \mathrm{mg}, 10.0 \mu \mathrm{mol})$ in $\mathrm{CH}_{3} \mathrm{NO}_{2}(0.4 \mathrm{~mL}$, $0.25 \mathrm{M}$ ) was stirred at $-20{ }^{\circ} \mathrm{C}$. The solution was concentrated and purified by flash chromatography $\left(\mathrm{Al}_{2} \mathrm{O}_{3}, 60 \%\right.$ ethyl acetate in hexanes) to furnish the product as a white solid $(20.1 \mathrm{mg}, 65 \%)$ which was determined to be $95 \%$ ee by chiral HPLC analysis $\left([\alpha]_{\mathrm{D}}\right.$ $=-14.8^{\circ}$ (c 1.0, acetone) Chiralcel ${ } \mathrm{AD}, 90: 10$ hexanes:i-PrOH, $1 \mathrm{~mL} / \mathrm{min}, \operatorname{tr}$ (major) $=$ 19.0min, $\operatorname{tr}($ minor $)=13.1 \mathrm{~min}) . \mathrm{Mp}=140-142{ }^{\circ} \mathrm{C} ; \mathrm{R}_{f}=0.38$ (80\% EtOAc/hexanes); IR (film) 3356, 2978, 1681, 1551, 1524, $1351 \mathrm{~cm}^{-1} ;{ }^{1} \mathrm{H}$ NMR (400 MHz, $\left.\mathrm{CDCl}_{3}\right) \delta 8.23-8.21$ $(\mathrm{m}, 2 \mathrm{H}), 7.70(\mathrm{~d}, J=7.8 \mathrm{~Hz}, 1 \mathrm{H}), 7.60(\mathrm{dd}, J=7.9,8.6 \mathrm{~Hz}, 1 \mathrm{H}), 5.61(\mathrm{~d}, J=7.5 \mathrm{~Hz}, 1 \mathrm{H})$, $5.50(\mathrm{~d}, J=5.2 \mathrm{~Hz}, 1 \mathrm{H}), 4.92(\mathrm{~m}, 1 \mathrm{H}), 4.80(\mathrm{dd}, J=13.0,4.3 \mathrm{~Hz}, 1 \mathrm{H}), 1.46(\mathrm{~s}, 9 \mathrm{H}) ;{ }^{13} \mathrm{C}$ NMR (100 MHz, $\mathrm{CDCl}_{3}$ ) ppm 154.9, 148.9, 139.6, 132.7, 130.5, 123.9, 121.6, 81.6, 78.6, 52.3, 28.5; HRMS (CI): Exact mass calcd for $\mathrm{C}_{13} \mathrm{H}_{17} \mathrm{~N}_{3} \mathrm{O}_{6}[\mathrm{M}]^{+}, 311.1117$. Found 311.1118 .

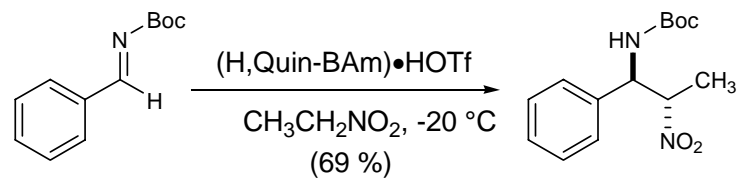

tert-Butyl (1R,2S)-2-nitro-1-phenylpropylcarbamate (3d). A solution of imine (100.0 $\mathrm{mg}, 487 \mu \mathrm{mol})$ and $(\mathrm{H}, \mathrm{Quin}-\mathrm{BAM}) \cdot \mathrm{TfOH}(25.3 \mathrm{mg}, 48.7 \mu \mathrm{mol})$ in $\mathrm{CH}_{3} \mathrm{CH}_{2} \mathrm{NO}_{2}(1.6 \mathrm{~mL}$, $0.25 \mathrm{M}$ ) was stirred at $-20{ }^{\circ} \mathrm{C}$. The solution was concentrated and purified by flash chromatography $\left(\mathrm{SiO}_{2}, 20 \%\right.$ ethyl acetate in hexanes) to furnish the product as a white solid (93.7 mg, $69 \%$ ) and a 14:1 mixture of diastereomers; the major diastereomer was determined to be 59\% ee by chiral HPLC analysis (Chiralcel ${ }_{\circledast}$ AD, 90:10 hexanes:i$\mathrm{PrOH}, 1 \mathrm{~mL} / \mathrm{min}, \operatorname{tr}_{\mathrm{r}}$ (major) $=10.1 \mathrm{~min}, \operatorname{tr}($ minor $\left.)=9.3 \mathrm{~min}\right) . \mathrm{Mp}=143-145^{\circ} \mathrm{C} ; \mathrm{R}_{f}=0.61$ (40\% EtOAc/hexanes); IR (film) 3383, 2975, 2938, 1684, $1544 \mathrm{~cm}^{-1} ;{ }^{1} \mathrm{H}$ NMR (400 $\left.\mathrm{MHz} \mathrm{CDCl}_{3}\right) \delta$ 7.28-7.32 (m, 3H), 7.20-7.23 (m, 2H), 5.35 (br s, 1H), 5.18 (dd, $J=8.5$, $6.1 \mathrm{~Hz}, 1 \mathrm{H}), 4.89$ (br s, $1 \mathrm{H}), 1.49(\mathrm{~d}, J=6.7 \mathrm{~Hz}, 3 \mathrm{H}), 1.39$ (s, 9H); ${ }^{13} \mathrm{C}$ NMR $(100 \mathrm{MHz}$, $\mathrm{CDCl}_{3}$ ) ppm 155.2, 136.7, 129.2, 128.8, 127.1, 86.0, 80.7, 57.7, 28.4, 15.5; HRMS (EI): Exact mass calcd for $\mathrm{C}_{14} \mathrm{H}_{21} \mathrm{~N}_{2} \mathrm{O}_{4}[\mathrm{M}+\mathrm{H}]^{+}, 281.1501$. Found 281.1491.

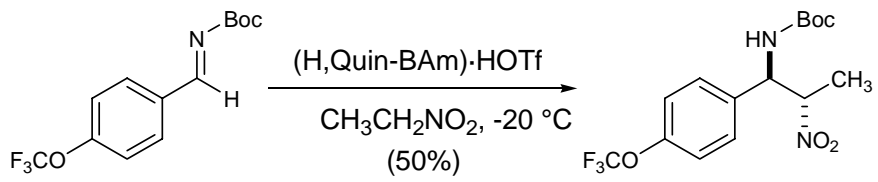


tert-Butyl (1R,2S)-2-nitro-1-(4-(trifluoromethoxy)phenyl)propylcarbamate (3e). A solution of the imine $(100.0 \mathrm{mg}, 346 \mu \mathrm{mol})$ and (H,Quin-BAM) ${ }^{\bullet} \mathrm{TfOH}(19.0 \mathrm{mg}, 34.5$ $\mu \mathrm{mol})$ in $\mathrm{CH}_{3} \mathrm{CH}_{2} \mathrm{NO}_{2}(1.6 \mathrm{~mL}, 0.25 \mathrm{M})$ was stirred at $-20{ }^{\circ} \mathrm{C}$. The solution was concentrated and purified by flash chromatography $\left(\mathrm{SiO}_{2}, 20 \%\right.$ ethyl acetate in hexanes) to furnish the product as a white solid $(62.5 \mathrm{mg}, 50 \%)$ and a 19:1 mixture of diastereomers; the major diastereomer was determined to be $81 \%$ ee by chiral HPLC analysis $\left([\alpha]_{\mathrm{D}}=-12.5^{\circ}\right.$ (c 1.0, acetone) Chiralcel $\mathbb{B} \mathrm{AD}, 95: 5$ hexanes:i-PrOH, $1 \mathrm{~mL} / \mathrm{min}$, $\operatorname{tr}($ major $)=19.7 \mathrm{~min}, \operatorname{tr}($ minor $)=12.1 \mathrm{~min}) . \mathrm{Mp}=113-115{ }^{\circ} \mathrm{C} ; \mathrm{R}_{f}=0.22(20 \%$ EtOAc/hexanes); IR (film) 3373, 2989, 2941, 1680, $1518 \mathrm{~cm}^{-1}$; ${ }^{1} \mathrm{H}$ NMR (400 MHz, $\left.\mathrm{CDCl}_{3}\right) \delta 7.26(\mathrm{~d}, J=8.2 \mathrm{~Hz}, 2 \mathrm{H}), 7.17(\mathrm{~d}, J=8.2 \mathrm{~Hz}, 2 \mathrm{H}), 5.35$ (br s, 1H), 5.17 (dd, $J=$ $8.5,5.8 \mathrm{~Hz}, 1 \mathrm{H}), 4.88(\mathrm{br} \mathrm{s}, 1 \mathrm{H}) 1.51(\mathrm{~d}, J=6.7 \mathrm{~Hz}, 3 \mathrm{H}), 1.39(\mathrm{~s}, 9 \mathrm{H}) ;{ }^{13} \mathrm{C}$ NMR $(125$ $\left.\mathrm{MHz}, \mathrm{CDCl}_{3}\right)$ ppm 155.0, 149.5, 148.3, 128.6, 121.5, 85.7, 81.0, 57.0, 28.4, 15.5; HRMS (EI): Exact mass calcd for $\mathrm{C}_{15} \mathrm{H}_{20} \mathrm{~F}_{3} \mathrm{~N}_{2} \mathrm{O}_{5}[\mathrm{M}+\mathrm{H}]^{+}$, 365.1324. Found 365.1315.

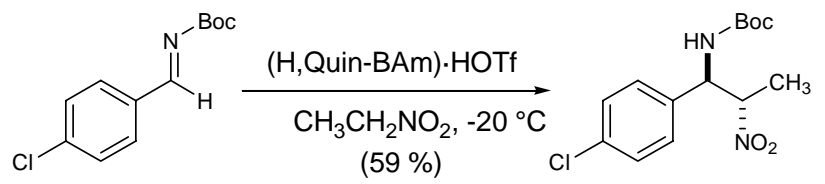

tert-Butyl (1R,2S)-1-(4-chlorophenyl)-2-nitropropylcarbamate (3f). A solution of the imine $(100.0 \mathrm{mg}, 417 \mu \mathrm{mol})$ and $(\mathrm{H}, \mathrm{Quin}-\mathrm{BAM}) \cdot \mathrm{TfOH}(21.6 \mathrm{mg}, 41.7 \mu \mathrm{mol})$ in $\mathrm{CH}_{3} \mathrm{CH}_{2} \mathrm{NO}_{2}(0.8 \mathrm{~mL}, 0.25 \mathrm{M})$ was stirred at $-20{ }^{\circ} \mathrm{C}$. The solution was concentrated and purified by flash chromatography $\left(\mathrm{SiO}_{2}, 20 \%\right.$ ethyl acetate in hexanes) to furnish the product as a white solid (77.8 $\mathrm{mg}, 59 \%)$ and a 17:1 mixture of diastereomers; the major diastereomer was determined to be $82 \%$ ee by chiral HPLC analysis $\left([\alpha]_{\mathrm{D}}=-25.4^{\circ}\right.$ (c 1.0, acetone), Chiralcel ${ }_{\circledast} \mathrm{AD}, 95: 5$ hexanes: $i-\mathrm{PrOH}, 1 \mathrm{~mL} / \mathrm{min}, \operatorname{tr}$ (major) $=25.5 \mathrm{~min}, \operatorname{tr}$ (minor) $=16.7 \mathrm{~min}$ ). $\mathrm{Mp}=142-144{ }^{\circ} \mathrm{C} ; \mathrm{R}_{f}=0.66$ (40\% EtOAc/hexanes); IR (film) 3393, 2981, 2935, 1682, $1524 \mathrm{~cm}^{-1}$; ${ }^{1} \mathrm{H}$ NMR (400 MHz, $\left.\mathrm{CDCl}_{3}\right) \delta 7.32(\mathrm{~d}, J=8.5 \mathrm{~Hz}, 2 \mathrm{H}), 7.18(\mathrm{~d}, J$ $=7.6 \mathrm{~Hz}, 2 \mathrm{H}), 5.39$ (br s, $1 \mathrm{H}), 5.15$ (dd, $J=8.9,5.8 \mathrm{~Hz}, 1 \mathrm{H}), 4.89$ (br s, $1 \mathrm{H}), 1.52$ (d, $J=$ $7.0 \mathrm{~Hz}, 3 \mathrm{H}), 1.42$ (s, 9H); ${ }^{13} \mathrm{C}$ NMR (100 MHz, $\mathrm{CDCl}_{3}$ ) ppm 155.0, 135.1, 134.8, 129.3, 128.5, 85.8, 81.0, 57.1, 28.4, 15.5; HRMS (EI): Exact mass calcd for $\mathrm{C}_{14} \mathrm{H}_{20} \mathrm{ClN}_{2} \mathrm{O}_{4}$ $[\mathrm{M}+\mathrm{H}]^{+}, 315.1112$. Found 315.1117.

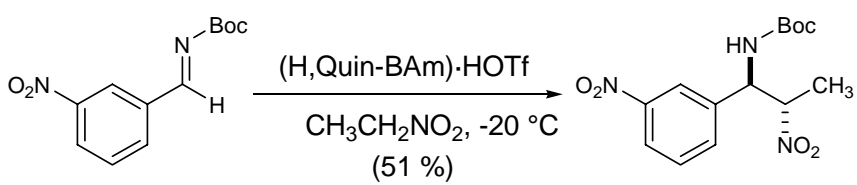

tert-Butyl (1R,2S)-2-nitro-1-(3-nitrophenyl)propylcarbamate (3g). A solution of imine $(100.0 \mathrm{mg}, 400 \mu \mathrm{mol})$ and $(\mathrm{H}, \mathrm{Quin}-\mathrm{BAM}) \cdot \mathrm{TfOH}(20.7 \mathrm{mg}, 39.9 \mu \mathrm{mol})$ in 
$\mathrm{CH}_{3} \mathrm{CH}_{2} \mathrm{NO}_{2}(1.6 \mathrm{~mL}, 0.25 \mathrm{M})$ was stirred at $-20{ }^{\circ} \mathrm{C}$. The solution was concentrated and purified by flash chromatography $\left(\mathrm{SiO}_{2}, 20 \%\right.$ ethyl acetate in hexanes) to furnish the product as a white solid (66.3 $\mathrm{mg}, 51 \%)$ and a 11:1 mixture of diastereomers; the major diastereomer was determined to be $89 \%$ ee by chiral HPLC analysis $\left([\alpha]_{\mathrm{D}}=-12.5^{\circ}\right.$ (c 1.0, acetone) Chiralcel ${ }_{\circledR} \mathrm{AD}, 90: 10$ hexanes:i-PrOH, $1 \mathrm{~mL} / \mathrm{min}, t_{\mathrm{r}}($ major$)=11.9 \mathrm{~min}$, $\operatorname{tr}(\operatorname{minor})=9.7 \mathrm{~min}) . \mathrm{Mp}=146-148{ }^{\circ} \mathrm{C} ; \mathrm{R}_{f}=0.61$ (40\% EtOAc/hexanes); IR (film) 3369, 2985, 1683, $1517 \mathrm{~cm}^{-1}$; ${ }^{1} \mathrm{H}$ NMR (400 MHz, $\left.\mathrm{CDCl}_{3}\right) \delta 8.20$ (ddd, $J=7.8,1.7,1.6 \mathrm{~Hz}$, $1 \mathrm{H}), 8.15$ (s, 1H), 7.62-7.54 (m, 2H), 5.52(br s, 1H), $5.30(\mathrm{dd}, \mathrm{J}=8.5,8.5 \mathrm{~Hz}, 1 \mathrm{H}), 4.95$ (br s, $1 \mathrm{H}), 1.58(\mathrm{~d}, J=6.8 \mathrm{~Hz}, 3 \mathrm{H}), 1.43(\mathrm{~s}, 9 \mathrm{H}) ;{ }^{13} \mathrm{C} \mathrm{NMR}\left(100 \mathrm{MHz}, \mathrm{CDCl}_{3}\right) \mathrm{ppm}$ 154.9, 148.7, 148.6, 133.4, 130.3, 123.9, 122.1, 85.5, 81.4, 57.0, 28.4, 15.5; HRMS (EI): Exact mass calcd for $\mathrm{C}_{14} \mathrm{H}_{19} \mathrm{~N}_{3} \mathrm{O}_{6}[\mathrm{M}]^{+}, 325.1274$. Found 325.1269.

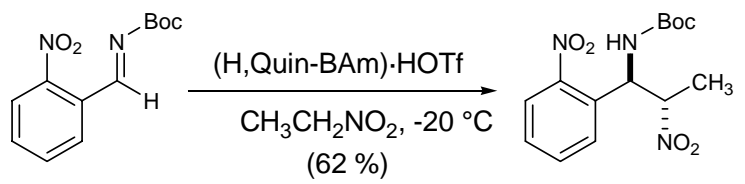

tert-Butyl (1R,2S)-2-nitro-1-(2-nitrophenyl)propylcarbamate (3h). A solution of imine $(50.0 \mathrm{mg}, 200 \mu \mathrm{mol})$ and (H,Quin-BAM) ${ }^{\circ} \mathrm{TfOH}(10.4 \mathrm{mg}, 20.1 \mu \mathrm{mol})$ in $\mathrm{CH}_{3} \mathrm{CH}_{2} \mathrm{NO}_{2}(0.8 \mathrm{~mL}, 0.25 \mathrm{M})$ was stirred at $-20{ }^{\circ} \mathrm{C}$. The solution was concentrated and purified by flash chromatography $\left(\mathrm{SiO}_{2}, 20 \%\right.$ ethyl acetate in hexanes) to furnish the product as a colorless oil (40.4 mg, $62 \%$ ) and a 7:1 mixture of diastereomers; the major diastereomer was determined to be $82 \%$ ee by chiral HPLC analysis $\left([\alpha]_{\mathrm{D}}=+55.0^{\circ}(c) 1.0\right.$, acetone) Chiralcel ${ }_{\circledast} \mathrm{OJ}, 90: 10$ hexanes:i-PrOH, $1 \mathrm{~mL} / \mathrm{min}, \operatorname{tr}$ (major) $=11.7 \mathrm{~min}, \operatorname{tr}$ (minor) $=13.4 \mathrm{~min}$ ). $\mathrm{R}_{f}=0.43$ (40\% EtOAc/hexanes); IR (film) 3340, 2980, 1703, $1553 \mathrm{~cm}^{-1} ;{ }^{1} \mathrm{H}$ NMR (400 MHz, $\left.\mathrm{CDCl}_{3}\right) \delta 8.06(\mathrm{~d}, J=7.6 \mathrm{~Hz}, 1 \mathrm{H}), 7.63(\mathrm{dd}, J=7.6,6.7 \mathrm{~Hz}, 1 \mathrm{H}), 7.53-$ 7.49 (m 2H), 5.74-5.66 (m, 2H), 5.24 (br s, 1H), 1.64 (d, J = 6.7 Hz, 3H), 1.40 (s, 9H); ${ }^{13} \mathrm{C}$ NMR $\left(125 \mathrm{MHz}, \mathrm{CDCl}_{3}\right) \mathrm{ppm} 155.1,148.4,134.6,132.2,130.1,129.7126 .4,84.6$, 81.1, 56.4, 28.5, 16.4; HRMS (EI): Exact mass calcd for $\mathrm{C}_{14} \mathrm{H}_{20} \mathrm{~N}_{3} \mathrm{O}_{6}[\mathrm{M}+\mathrm{H}]^{+}, 326.1352$. Found 326.1357.

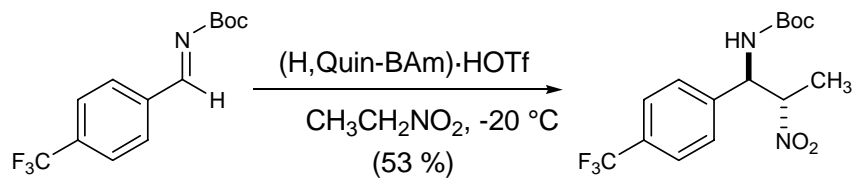


tert-Butyl (1R,2S)-1-(4-(trifluoromethyl)phenyl)-2-nitropropylcarbamate (3i). A solution of imine $(100.0 \mathrm{mg}, 366 \mu \mathrm{mol})$ and (H,Quin-BAM) $\mathrm{TfOH}(19.0 \mathrm{mg}, 36.6 \mu \mathrm{mol})$ in $\mathrm{CH}_{3} \mathrm{CH}_{2} \mathrm{NO}_{2}(1.6 \mathrm{~mL}, 0.25 \mathrm{M})$ was stirred at $-20{ }^{\circ} \mathrm{C}$. The solution was concentrated and purified by flash chromatography $\left(\mathrm{SiO}_{2}, 20 \%\right.$ ethyl acetate in hexanes) to furnish the product as a white solid (67.5 mg, $53 \%$ ) and a 19:1 mixture of diastereomers; the major diastereomer was determined to be $84 \%$ ee by chiral HPLC analysis $\left([\alpha]_{\mathrm{D}}=-11.8^{\circ}\right.$ (c 1.0, acetone) Chiralcel ${ }$ OD, 90:10 hexanes:i-PrOH, $1 \mathrm{~mL} / \mathrm{min}, \operatorname{tr}($ major $)=11.8 \mathrm{~min}$, $\operatorname{tr}($ minor $)=7.6 \mathrm{~min}) . \mathrm{Mp}=137-139{ }^{\circ} \mathrm{C} ; \mathrm{R}_{f}=0.65$ (40\% EtOAc/hexanes); IR (film) 3373, 2987, 1682, $1544 \mathrm{~cm}^{-1} ;{ }^{1} \mathrm{H}$ NMR $\left(400 \mathrm{MHz}, \mathrm{CDCl}_{3}\right) \delta 7.63(\mathrm{~d}, J=8.2 \mathrm{~Hz}, 2 \mathrm{H}), 7.37(\mathrm{~d}, J$ $=8.2 \mathrm{~Hz}, 2 \mathrm{H}), 5.39$ (br s, $1 \mathrm{H}), 5.24$ (dd, $J=8.5,5.8 \mathrm{~Hz}, 1 \mathrm{H}), 4.93$ (br s, $1 \mathrm{H}), 1.54$ (d, $J=$ $6.7 \mathrm{~Hz}, 3 \mathrm{H}), 1.43$ (s, 9H); ${ }^{13} \mathrm{C}$ NMR (125 MHz, $\mathrm{CDCl}_{3}$ ) ppm 155.0, 140.1, 131.1, 127.6, 157.4, 122.6, 85.6, 57.2, 28.4, 15.4; HRMS (EI): Exact mass calcd for $\mathrm{C}_{15} \mathrm{H}_{20} \mathrm{~F}_{3} \mathrm{~N}_{2} \mathrm{O}_{4}$ $[\mathrm{M}+\mathrm{H}]^{+}, 349.1375$. Found 349.1375.

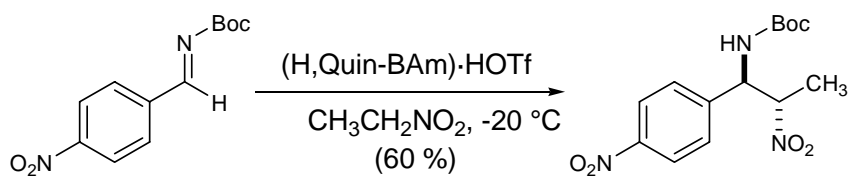

tert-Butyl (1R,2S)-2-nitro-1-(4-nitrophenyl)propylcarbamate (3j). A solution of imine (50.0 mg, $200 \mu \mathrm{mol})$ and (H,Quin-BAM) ${ }^{-T f O H}(10.4 \mathrm{mg}, 20.1 \mu \mathrm{mol})$ in $\mathrm{CH}_{3} \mathrm{CH}_{2} \mathrm{NO}_{2}$ $(0.4 \mathrm{~mL}, 0.25 \mathrm{M})$ was stirred at $-20^{\circ} \mathrm{C}$. The solution was concentrated and purified by flash chromatography $\left(\mathrm{SiO}_{2}, 20 \%\right.$ ethyl acetate in hexanes) to furnish the product as a yellow oil (38.9 mg, $60 \%$ ) and a 7:1 mixture of diastereomers; the major diastereomer was determined to be $90 \%$ ee by chiral HPLC analysis $\left([\alpha]_{\mathrm{D}}=-7.4^{\circ}\right.$ (c 1.0, acetone) Chiralcel ${ }_{\circledast} \mathrm{AD}, 80: 20$ hexanes:i-PrOH, $1 \mathrm{~mL} / \mathrm{min}, \operatorname{tr}$ (major) $=13.1 \mathrm{~min}, \operatorname{tr}($ minor $)=7.5$ $\min$ ). $\mathrm{R}_{f}=0.60$ (40\% EtOAc/hexanes); IR (film) 3385, 2981, 2935, 1699, $1557 \mathrm{~cm}^{-1} ;{ }^{1} \mathrm{H}$ NMR (400 MHz, $\left.\mathrm{CDCl}_{3}\right) \delta 8.23(\mathrm{~d}, J=8.7 \mathrm{~Hz}, 2 \mathrm{H}), 7.44(\mathrm{~d}, J=8.7 \mathrm{~Hz}, 2 \mathrm{H}), 5.49$ (br s, 1H), 5.27 (dd, $J=7.1,6.2 \mathrm{~Hz}, 1 \mathrm{H}), 4.94$ (br s, 1H), 1.55 (d, $J=6.7 \mathrm{~Hz}, 3 \mathrm{H}), 1.43$ (s, 9H); ${ }^{13} \mathrm{C}$ NMR (125 MHz, $\mathrm{CDCl}_{3}$ ) ppm 154.9, 148.3, 128.3, 124.4, 123.7, 85.4, 81.4, 57.2, 28.4, 18.3; HRMS (EI): Exact mass calcd for $\mathrm{C}_{14} \mathrm{H}_{20} \mathrm{~N}_{3} \mathrm{O}_{6}[\mathrm{M}+\mathrm{H}]^{+}, 326.1352$. Found 326.1351 .

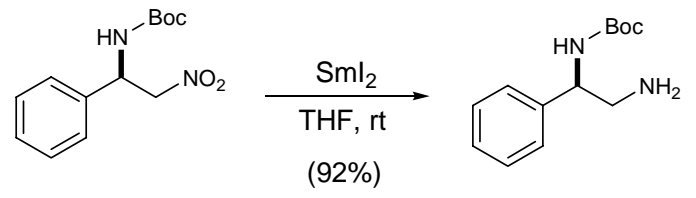


tert-Butyl (R)-2-amino-1-phenylethylcarbamate (S2). A solution of 1,2-diodoethane (137.6 mg, $488 \mu \mathrm{mol})$ in THF $(1 \mathrm{~mL})$ was added dropwise to $\mathrm{Sm}^{0}$ powder $(79.1 \mathrm{mg}, 526$ $\mu \mathrm{mol})$ in a flame-dried, argon purged flask. The suspension was stirred for an additional $2 \mathrm{~h}$ at $\mathrm{rt}$ (a deep blue color emerged), then a solution of the nitroalkyl (20.0 $\mathrm{mg}, 75.1$ $\mu \mathrm{mol})$ in $\mathrm{MeOH} / \mathrm{H}_{2} \mathrm{O}(1 \mathrm{~mL}, 1: 1)$ was added. After $4 \mathrm{~h}$, a solution of oxalic acid (133 $\mathrm{mg})$ in $\mathrm{H}_{2} \mathrm{O}(2 \mathrm{~mL})$ was added which immediately formed a brown precipitate. Filtration and addition of $1 \mathrm{M} \mathrm{NaOH}(1 \mathrm{~mL})$ followed by extraction with ethyl acetate, drying with $\mathrm{Na}_{2} \mathrm{SO}_{4}$ and concentration gave the product as a yellow oil $(16.3 \mathrm{mg}, 92 \%)$. The optical rotation $[\alpha]_{\mathrm{D}}=-16.8^{\circ}\left(\mathrm{c} 0.6, \mathrm{CH}_{3} \mathrm{Cl}\right)$ is consistent with the $(R)$-enantiomer reported previously. 5

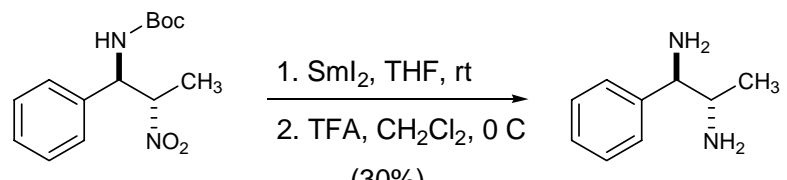

$(30 \%)$

(1R,2S)-1-phenylpropane-1,2-diamine (S3). A solution of 1,2-diodoethane (522.9 mg, $1.86 \mathrm{mmol})$ in THF (3 mL) was added dropwise to $\mathrm{Sm}^{0}$ powder $(300.4 \mathrm{mg}, 2.0 \mathrm{mmol})$ in a flame-dried, argon purged round bottom flask. The suspension was stirred for an additional $2 \mathrm{~h}$ at $\mathrm{rt}$ (a deep blue color emerged), then a solution of the nitroalkane (80.0 $\mathrm{mg}, 285 \mu \mathrm{mol})$ in $\mathrm{MeOH} / \mathrm{H}_{2} \mathrm{O}(3 \mathrm{~mL}, 1: 1)$ was added. After $4 \mathrm{~h}$, a solution of oxalic acid (400 mg) in $\mathrm{H}_{2} \mathrm{O}(2 \mathrm{~mL})$ was added which immediately formed a brown precipitate. Filtration and addition of $1 \mathrm{M} \mathrm{NaOH}(4 \mathrm{~mL})$ followed by extraction with ethyl acetate, drying with $\mathrm{Na}_{2} \mathrm{SO}_{4}$, and concentration gave the Boc protected diamine as a yellow oil. The oil was dissolved in $\mathrm{CH}_{2} \mathrm{Cl}_{2}$, cooled to $0{ }^{\circ} \mathrm{C}$, and TFA $(0.2 \mathrm{~mL})$ was added. The solution was allowed to warm to rt overnight, followed by addition of $1 \mathrm{M} \mathrm{NaOH}(5 \mathrm{ml})$. After extraction with $\mathrm{CH}_{2} \mathrm{Cl}_{2}$, the combined organic layers were dried and concentrated to give the product diamine as a yellow oil $\left(12.7 \mathrm{mg}, 30 \%\right.$ over two steps). The ${ }^{1} \mathrm{H} \mathrm{NMR}$ spectrum and optical rotation $\left([\alpha]_{\mathrm{D}}=+7.4^{\circ}(\mathrm{c} 1.0,1 \mathrm{M} \mathrm{HCl})\right)$ were consistent with the configuration shown based on the literature values. ${ }^{6}$

\footnotetext{
${ }^{5}$ O’Brien, P. M.; Sliskovic, D. R.; Blankley, C. J.; Roth, B. D.; Wilson, M. W.; Hamelehle, K. L.; Krause, B. R.; Stanfield, R. L. J. Med. Chem. 1994, 37, 1810-1822.

${ }^{6}$ Gust, R.; Gelbcke, M.; Angermaier, B.; Bachmann, H.; Krauser, R; Schoneneberger, H. Inorganica Chimica Acta 1997, 264, 145-160.
} 